\title{
Strong parity vertex coloring of plane graphs*
}

\author{
Tomáš Kaiser ${ }^{\dagger}$ Ondřej Rucký ${ }^{\ddagger} \quad$ Matěj Stehlík $k^{\S} \quad$ Riste Škrekovski ${ }^{\Uparrow}$
}

\begin{abstract}
A strong parity vertex coloring of a 2-connected plane graph is a coloring of the vertices such that every face is incident with zero or an odd number of vertices of each color. We prove that every 2-connected loopless plane graph has a strong parity vertex coloring with 97 colors. Moreover the coloring we construct is proper. This proves a conjecture of Czap and Jendrol' [Discuss. Math. Graph Theory 29 (2009), pp. 521-543.]. We also provide examples showing that eight colors may be necessary (ten when restricted to proper colorings).
\end{abstract}

Keywords: graph, strong parity vertex coloring, strong parity chromatic number, proper coloring, face, discharging

\section{Introduction}

The notions of strong parity vertex coloring and the strong parity chromatic number were defined by Czap and Jendrol' [3]. Let us recall their definition in an equivalent form. Let $G$ be a nontrivial connected plane graph, and let $f$ be one of its faces. (Throughout the paper, graphs are allowed to have parallel edges but no loops.) Consider a (possibly improper) vertex coloring of $G$. The face $f$ satisfies the strong parity vertex coloring condition (spvcondition for short) with respect to the coloring if for each color $c$ of the coloring, there is

${ }^{*}$ This work was supported by the Czech-Slovenian bilateral research project MEB 091037 and projects 201/09/0197 and P202/12/G061 of the Czech Science Foundation.

$\dagger$ Department of Mathematics, Institute for Theoretical Computer Science (CE-ITI) and European Centre of Excellence NTIS-New Technologies for Information Society, University of West Bohemia, Univerzitní 8, 30614 Plzeň, Czech Republic. E-mail: kaisert@kma.zcu.cz.

${ }^{\ddagger}$ Former affiliation: Department of Mathematics, University of West Bohemia, Univerzitní 8, 30614 Plzeň, Czech Republic.

$\S$ UJF-Grenoble 1 / CNRS / Grenoble-INP, G-SCOP UMR5272 Grenoble, F-38031, France. E-mail: matej.stehlik@g-scop.inpg.fr.

IDepartment of Mathematics, University of Ljubljana, Ljubljana \& Faculty of Information Studies, Novo Mesto \& FAMNIT, University of Primorska, Koper, Slovenia. E-mail: skrekovski@gmail.com. Partially supported by Slovenian ARRS Program P1-00383 and Creative Core - FISNM - 3330-13-500033. 
zero or an odd number of occurrences of vertices colored with $c$ on a closed facial walk of $f$. The coloring is a strong parity vertex coloring (spv-coloring for short) if the spv-condition holds for every face of $G$. Assume now that $G$ is 2-connected. Then the minimum number of colors in an spv-coloring of $G$ is called the strong parity chromatic number of $G$ and is denoted by $\chi_{s}(G)$. The restriction to 2-connected graphs in the definition of $\chi_{s}$ is essential, since there are plane graphs of connectivity one that do not admit any spv-coloring (an example of Czap and Jendrol' [3] consists of two triangles sharing one vertex). Similarly, the existence of an spv-coloring cannot be guaranteed for graphs with loops (which is why we exclude them in our definition).

Czap and Jendrol' [3] conjectured that there is a constant bound $K$ on $\chi_{s}$ in the class of 2-connected plane graphs. Furthermore, they suggested that the best possible bound equals 6 , providing an infinite family of graphs with $\chi_{s}=6$. The main result of our paper confirms the conjecture for the class of 2-connected plane graphs with an added restriction to proper colorings:

Theorem 1.1. Every 2-connected plane (loopless) graph has a proper spv-coloring with at most 97 colors.

The proof is given in Section 2. (During the preparation of this paper, another proofgiving a slightly worse constant — was independently found by Czap, Jendrol', and Voigt [4].) In Section 3, we present examples showing that the best possible value of $K$ in the above conjecture is at least 8 , or at least 10 with the restriction to proper colorings.

It should be noted that prior to the introduction of parity vertex colorings, a related type of edge coloring was considered in $[1,2]$. An edge variant of parity coloring (called facial parity edge coloring) was recently studied, e.g., in [5].

In the remainder of this section, we establish the basic notation used throughout the paper; the notions not mentioned here are standard in graph theory [6]. As mentioned above, graphs are assumed to be loopless, but parallel edges are allowed. A graph is called trivial if it is empty or consists of a single vertex. Let $G$ be a plane graph; let $v$ be a vertex, $e_{1}$ and $e_{2}$ edges, and $f$ a face of $G$. Then $F(v)$ or $F\left(e_{1}\right)$ denotes the set of faces incident with $v$ and $e_{1}$ respectively. The boundary vertices and boundary edges of $f$ are all the vertices and edges of $G$, respectively, incident with $f$. The sets of these vertices and edges are denoted by $V(f)$ and $E(f)$ respectively. We refer to $|V(f)|$ as the length of $f$.

The degree of $v$ in $G$, i.e., the number of edges of $G$ incident with $v$, is denoted by $d(v)$. If $d(v)=2$, the vertex $v$ is a 2-vertex; when $d(v)>2$, we call $v$ a high-degree vertex or a vertex of high degree. The edges $e_{1}$ and $e_{2}$ are parallel if they are not loops and share their end-vertices. When $e_{1}$ and $e_{2}$ are parallel and constitute the boundary of $f$, the face $f$ is called a digon. A path $P$ is trivial if it comprises a single vertex, that is, the length of $P$ equals 0 . Every vertex of $P$ other than its end-vertices is called an internal vertex of $P$. Finally, we remark that for all the notation defined, the relevant graph may be referred to by a subscript whenever necessary. For example, we write $F_{G}(v)$ or $d_{G}(v)$ if this graph is $G$. 


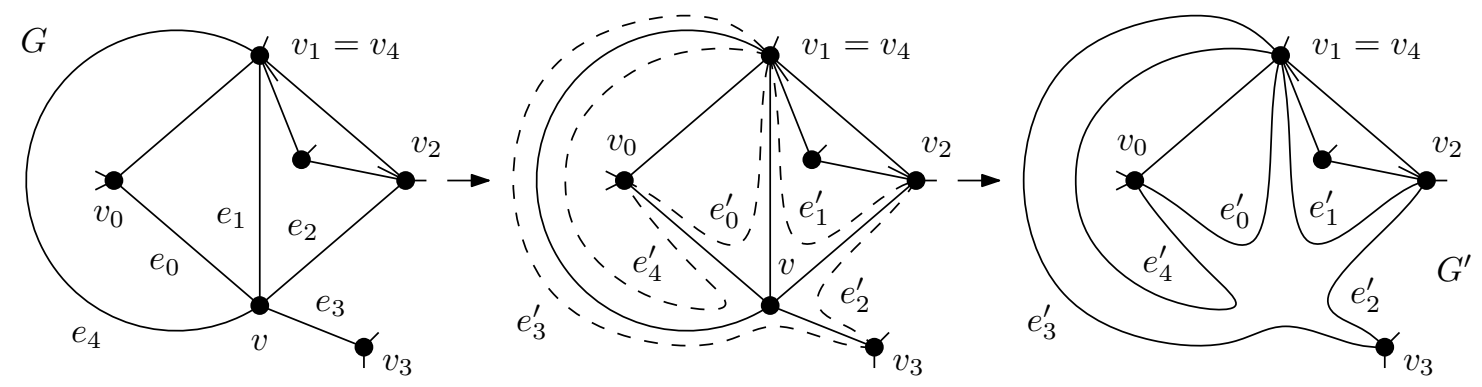

Figure 2.1. The annihilation of a vertex $v$. The original graph $G$ is on the left, the resulting graph $G^{\prime}$ on the right.

\section{Upper bound}

This section is devoted to the proof of the main result, Theorem 1.1. In Section 2.1, we prove certain structural properties of a minimal counterexample. These are used in an application of the discharging method in Section 2.2.

We now introduce a graph operation to be used in the proof of Lemma 2.4. Let $G$ be a plane graph and $v \in V(G)$ a vertex of degree $d \geq 2$, and let the edges incident with $v$ be enumerated in a clockwise order as $e_{i}=v v_{i}, i \in \mathbb{Z}_{d}$ (we write $\mathbb{Z}_{d}$ for the set $\{0, \ldots, d-1\}$ with addition modulo $d$ ). Suppose that the vertices $v_{i}$ are pairwise different (that is, $v$ is not incident with a pair of parallel edges). The annihilation of $v$ is the construction of a plane graph $G^{\prime}$ from $G$ defined as follows:

(1) add edges $e_{i}^{\prime}=v_{i} v_{i+1}, i \in \mathbb{Z}_{d}$, embedded in the plane so that for each $i$, the edges $e_{i}$, $e_{i+1}$, and $e_{i}^{\prime}$, in this order, constitute a facial walk;

(2) delete $v$ together with all the edges $e_{i}$.

Intuitively, one may achieve the desired embeddings of the edges $e_{i}^{\prime}$ by drawing each $e_{i}^{\prime}$ 'close enough' to the curve consisting of the embeddings of $e_{i}$ and $e_{i+1}$; see Figure 2.1 for an example of a properly conducted annihilation.

Regarding the faces of $G$ and $G^{\prime}$, it is obvious that the following holds:

Observation 2.1. Let $G^{\prime}$ be obtained from $G$ by the annihilation of a vertex $v \in V(G)$. Then

(1) every face of $G$ not in $F_{G}(v)$ is also a face of $G^{\prime}$;

(2) each face $g \in F_{G}(v)$ has its counterpart $g^{\prime}$ in $G^{\prime}$ such that a facial walk of $g^{\prime}$ may be obtained from a facial walk of $g$ by replacing each of its subsequences of the form $e_{i} v e_{i+1}$ with $e_{i}^{\prime}$, and hence $V\left(g^{\prime}\right)=V(g)-\{v\}$;

(3) there is precisely one more face in $G^{\prime}$, having the sequence $v_{0} e_{d-1}^{\prime} v_{d-1} e_{d-2}^{\prime} \ldots v_{1} e_{0}^{\prime} v_{0}$ as its facial walk. 
The assumption that $v$ is not incident with a pair of parallel edges is essential: without it, the annihilation of $v$ may produce a loop as well as a cutvertex. On the other hand, the following lemma shows that parallel edges incident with $v$ are the only reason for such a result:

Lemma 2.2. Let $v$ be a vertex of a 2-connected (loopless) plane graph $G,|V(G)| \geq 4$, such that $v$ is incident with no pair of parallel edges. Then the graph $G^{\prime}$ obtained from $G$ by annihilating $v$ is 2-connected (and loopless).

Proof. We use the well-known fact that a connected loopless plane graph $G$ on at least three vertices is 2-connected if and only if the facial walk of each of its faces is a cycle. (The 'only if' direction is Proposition 4.2.6 in [6]. Conversely, for any cutvertex $v$ of $G$ there is a face whose boundary contains two neighbors of $v$ in different components of $G-v$. Since each cycle is contained within some block, the boundary of this face cannot be a cycle.)

We use this criterion for both $G$ and $G^{\prime}$ in the following. (Note that $G^{\prime}$ is connected.) Take an arbitrary face $f^{\prime}$ of $G^{\prime}$, and let $W$ be a facial walk of $f^{\prime}$. We may assume that $f^{\prime}$ is not a face of $G$, otherwise there is nothing to show. Thus by Observation 2.1, $W$ is either the walk $v_{0} e_{d-1}^{\prime} v_{d-1} e_{d-2}^{\prime} \ldots v_{1} e_{0}^{\prime} v_{0}$ (up to the choice of the end-vertex), or it arises from a facial walk of a face of $G$ by replacing each of its subsequences of the form $e_{i} v e_{i+1}$ with $e_{i}^{\prime}$. In both cases, it follows from the assumptions that $W$ is a cycle.

Finally, we include a technical lemma that will greatly simplify the case analysis in the proof of Claim 1 in Section 2.2.

Lemma 2.3. Let $\left(l_{i}\right),\left(l_{i}^{\prime}\right), i=0, \ldots, k$, be tuples of positive integers such that $l_{j} \leq l_{j}^{\prime}$ for every $j \neq k$, and $l_{k}^{\prime} \geq l_{j}^{\prime}$ for every $j \neq k$ with $l_{j}<l_{j}^{\prime}$. Then $\sum_{i=0}^{k} l_{i}^{\prime} \leq \sum_{i=0}^{k} l_{i}$ or $\sum_{i=0}^{k} 1 / l_{i}^{\prime} \leq \sum_{i=0}^{k} 1 / l_{i}$.

Proof. We first prove that if $\sum_{i=0}^{k} l_{i}^{\prime}=\sum_{i=0}^{k} l_{i}$ and the tuples are distinct, then $\sum_{i=0}^{k} 1 / l_{i}^{\prime}<$ $\sum_{i=0}^{k} 1 / l_{i}$. We proceed by induction on the size of the set $J:=\left\{j: j \neq k, l_{j}<l_{j}^{\prime}\right\}$. From the assumption that $\left(l_{i}^{\prime}\right)$ and $\left(l_{i}\right)$ are distinct and have the same sum (and $l_{j} \leq l_{j}^{\prime}$ for $j \neq k$ ), it follows that $J$ is nonempty. Now, fix $j_{0}$ as some index in $J$, and let $d:=l_{j_{0}}^{\prime}-l_{j_{0}}$. Consider a tuple $\left(l_{i}^{\prime \prime}\right)$ such that $l_{j_{0}}^{\prime \prime}=l_{j_{0}}^{\prime}=l_{j_{0}}+d, l_{k}^{\prime \prime}=l_{k}-d$, and $l_{j}^{\prime \prime}=l_{j}$ for each remaining index $j$. Clearly, $\sum_{i=0}^{k} l_{i}^{\prime \prime}=\sum_{i=0}^{k} l_{i}^{\prime}, l_{k}^{\prime \prime} \geq l_{k}^{\prime}$, and the number of $j \neq k$ such that $l_{j}^{\prime \prime} \neq l_{j}^{\prime}$ equals $|J|-1$. We have

$$
\sum_{i=0}^{k} \frac{1}{l_{i}^{\prime \prime}}=\sum_{i=0}^{k} \frac{1}{l_{i}}+\left(\frac{1}{l_{j_{0}}^{\prime \prime}}-\frac{1}{l_{j_{0}}}\right)+\left(\frac{1}{l_{k}^{\prime \prime}}-\frac{1}{l_{k}}\right)=\sum_{i=0}^{k} \frac{1}{l_{i}}-d\left(\frac{1}{l_{j_{0}}\left(l_{j_{0}}+d\right)}-\frac{1}{l_{k}^{\prime \prime}\left(l_{k}^{\prime \prime}+d\right)}\right),
$$

and since

$$
l_{k}^{\prime \prime} \geq l_{k}^{\prime} \geq l_{j_{0}}^{\prime}>l_{j_{0}}
$$

by the assumptions and the choice of $j_{0}$, it follows immediately that

$$
\sum_{i=0}^{k} \frac{1}{l_{i}^{\prime \prime}}<\sum_{i=0}^{k} \frac{1}{l_{i}}
$$


When $|J|=1$ (the base case of the induction), the tuple $\left(l_{i}^{\prime \prime}\right)$ equals $\left(l_{i}^{\prime}\right)$ and there is nothing more to prove. Otherwise, we may apply the induction hypothesis to $\left(l_{i}^{\prime \prime}\right)$ and $\left(l_{i}^{\prime}\right)$, in this order, obtaining $\sum_{i=0}^{k} 1 / l_{i}^{\prime}<\sum_{i=0}^{k} 1 / l_{i}^{\prime \prime}$; this together with (2.1) gives the desired conclusion.

Now we prove the lemma. We may suppose that $\sum_{i=0}^{k} l_{i}^{\prime}>\sum_{i=0}^{k} l_{i}$. If $l_{k}^{\prime} \geq l_{k}$, then trivially $\sum_{i=0}^{k} 1 / l_{i}^{\prime}<\sum_{i=0}^{k} 1 / l_{i}$. Otherwise, we claim that there exists a tuple $\left(l_{i}^{\prime \prime}\right)$ such that $l_{j} \leq l_{j}^{\prime \prime} \leq l_{j}^{\prime}$ for every $j \neq k, l_{k}^{\prime \prime}=l_{k}^{\prime}$, and $\sum_{i=0}^{k} l_{i}^{\prime \prime}=\sum_{i=0}^{k} l_{i}$. Indeed, $\left(l_{i}^{\prime \prime}\right)$ may be obtained from $\left(l_{i}^{\prime}\right)$ by replacing some of the values $l_{j}^{\prime}(j<k)$ with smaller ones, using the fact that $\sum_{i=0}^{k-1}\left(l_{i}^{\prime}-l_{i}\right)>l_{k}-l_{k}^{\prime}$. Note that $\left(l_{i}^{\prime \prime}\right)$ is distinct from both $\left(l_{i}\right)$ and $\left(l_{i}^{\prime}\right)$. It follows from the above that $\sum_{i=0}^{k} 1 / l_{i}^{\prime \prime}<\sum_{i=0}^{k} 1 / l_{i}$. Furthermore, since $l_{i}^{\prime \prime} \leq l_{i}^{\prime}$ for all $i \leq k$ and the tuples are not equal, $\sum_{i=0}^{k} 1 / l_{i}^{\prime}<\sum_{i=0}^{k} 1 / l_{i}^{\prime \prime}$. The proof is now complete.

\subsection{Reducibility}

Let $G$ be a counterexample to Theorem 1.1 with the minimum number of vertices, and subject to this condition, with the minimum number of edges.

In Lemma 2.4 below, we infer several constraints applying to $G$. Based on these constraints, we derive bounds for the (reduced) face degree of a vertex in $G$ in Lemma 2.5. As per standard terminology, a graph contradicting Lemma 2.4 is said to be reducible.

Before stating the lemma, we introduce some terminology. Let $v$ be a vertex and $f$ a face of $G$. The face-vertex neighborhood of $v$ in $G$, denoted by $N^{F}(v)$, is defined as $\left(\bigcup_{g \in F(v)} V(g)\right)-\{v\}$. Similarly, the f-reduced face-vertex neighborhood of $v$, referred to as $N^{F}(v, f)$, is the set $\left(\bigcup_{g \in F(v), g \neq f} V(g)\right)-\{v\}$. We call the sizes of these sets the face degree of $v$ and $f$-reduced face degree of $v$ respectively, writing $d^{F}(v)$ and $d^{F}(v, f)$ respectively. As with the other notation, the graph $G$ is included as a subscript if necessary. For instance, we may write $N_{G}^{F}(v)$ or $d_{G}^{F}(v, f)$.

Lemma 2.4. The graph $G$ has the following properties:

(1) $|V(G)|>97$

(2) $G$ does not contain parallel edges; in particular, $G$ is without digons;

(3) no facial walk of a face of $G$ contains four consecutive 2-vertices;

(4) for every vertex $v$ of $G$, it holds $d^{F}(v) \geq 97$;

(5) for every two vertices $u$ and $v$ of $G$ such that $F(u) \cap F(v)=\{f\}$, it holds $d^{F}(u, f)+$ $d^{F}(v, f) \geq 96$.

Proof. By assumption, $G$ is a 2-connected graph. We prove each of the assertions by contradiction. To see (1), consider an assignment of a different color to each vertex of $G$.

We proceed to show assertion (2). Let $e_{1}$ and $e_{2}$ denote two parallel edges in $G$. We distinguish two cases. If $e_{1}$ together with $e_{2}$ delimit a digon $f$, we simply delete one of the 
two edges, say $e_{1}$, obtaining a (2-connected) graph $G^{\prime}$. By the minimality of $G$, the graph $G^{\prime}$ has a proper spv-coloring $c$ with at most 97 colors. As all faces of $G$ except $f$ have their counterparts in $G^{\prime}$ with the same sets of boundary vertices, and $c$ is proper, $c$ is also a proper spv-coloring of $G$.

When, on the other hand, the curve $\mathcal{C}$ comprising the embeddings of $e_{1}$ and $e_{2}$ is not the boundary of a digon, we produce two graphs $G_{1}$ and $G_{2}$ by deleting the interior and exterior of $\mathcal{C}$ from $G$, respectively. Both these graphs are clearly 2-connected (in particular, each has at least three vertices), and smaller than $G$ with respect to the given ordering; thus each has a proper spv-colorings with at most 97 colors. The colorings can be chosen so that they coincide at the common vertices, i.e., the end-vertices of $e_{1}$, and the number of colors in their union $c$ is minimal. Then $c$ is a proper spv-coloring of $G$ using at most 97 colors.

Next, suppose $x_{1} x_{2} x_{3} x_{4}$ is a path contradicting statement (3). Let $v_{1}$ be the neighbor of $x_{1}$ in $G$ other than $x_{2}$, and let $v_{4}$ be the neighbor of $x_{4}$ other than $x_{3}$. The vertices $v_{1}$ and $v_{4}$ are distinct and different from all $x_{i}, i=1, \ldots, 4$, otherwise the facial walk would contain just four or five vertices, and by the 2-connectedness of $G$ these would be the only vertices of $G$; a contradiction to assertion (1). We construct a graph $G^{\prime}$ by contracting the path $x_{1} \ldots x_{4} v_{4}$ into a single vertex $v_{4}^{\prime}$. It remains 2 -connected due to statement (1), and hence by assumption, $G^{\prime}$ has a proper spv-coloring $c$ with at most 97 colors. As $v_{1}$ and $v_{4}^{\prime}$ are adjacent in $G^{\prime}$, we obtain $c\left(v_{1}\right) \neq c\left(v_{4}^{\prime}\right)$. Now, we use the coloring for the corresponding vertices of $G$ and assign the color $c\left(v_{1}\right)$ to $x_{2}, x_{4}$, and the color $c\left(v_{4}^{\prime}\right)$ to $x_{1}, x_{3}, v_{4}$. This way the occurrence of the colors $c\left(v_{1}\right)$ and $c\left(v_{4}^{\prime}\right)$ preserves the parity on the corresponding facial walks, and we obtain a proper spv-coloring of $G$ with no more than 97 colors.

Now we focus on assertion (4). Suppose it does not hold. We perform the annihilation of $v$, obtaining a graph $G^{\prime}$. By parts (1) and (2), $G$ has obviously more than 3 vertices and has no parallel edges incident with $v$, and since $G$ is 2-connected, Lemma 2.2 assures that $G^{\prime}$ is 2-connected, as well. By the minimality of $G, G^{\prime}$ has a proper spv-coloring $c$ with at most 97 colors. Using $c$ for $G$ and assigning to $v$ a color not used by $c$ on any vertex in $N_{G}^{F}(v)$, but if possible present in $c$, we obtain a proper coloring of $G$ of cardinality less than or equal to 97.

By Observation 2.1, the only faces of $G$ whose sets of boundary vertices differ from those of their counterparts in $G^{\prime}$ are the elements of $F_{G}(v)$, but by the choice of the color of $v$, the spv-condition is maintained for them. Hence, the coloring of $G$ is also an spv-coloring; a contradiction.

Finally we deal with statement (5). Suppose it does not hold. We construct a graph $G^{\prime \prime}$ by annihilating $u$. As above, $G^{\prime \prime}$ is 2-connected, and hence we may annihilate $v$ in $G^{\prime \prime}$ to obtain the graph $G^{\prime}$. Since $u$ and $v$ have precisely one common incident face in $G$, they are not adjacent; therefore, the annihilation of $u$ does not create any new edges at $v$. This means, by part (2), that there is no pair of parallel edges incident with $v$ in $G^{\prime \prime}$, and thus, considering statement (1) again, Lemma 2.2 can be applied to the annihilation of $v$. We conclude that $G^{\prime}$ is 2-connected. As it is also smaller than $G$ with respect to our order, there is a proper spv-coloring $c^{\prime}$ of $G^{\prime}$ using at most 97 colors.

We extend the coloring to $G$ as follows. If there exists a color used by $c^{\prime}$ on a vertex in 
$V_{G}(f)-N_{G}^{F}(u, f)-N_{G}^{F}(v, f)$ and on no vertex in $N_{G}^{F}(u, f) \cup N_{G}^{F}(v, f)$, we assign this color to both $u$ and $v$. Otherwise we color each of $u$ and $v$ with a different color not used by $c^{\prime}$ on any vertex in $N_{G}^{F}(u, f) \cup N_{G}^{F}(v, f)$, but if possible appearing in $c^{\prime}$. Either case yields a coloring $c$ of $G$ with no more than 97 colors.

For the desired contradiction, it remains to show that $c$ is a proper spv-coloring of $G$. As the neighbors of $u$ and $v$ belong to $N_{G}^{F}(u, f) \cup N_{G}^{F}(v, f)$, the coloring is indeed proper. Next, by Observation 2.1 and the assumption about $F(u) \cap F(v)$, we see that each face $g$ of $G$ has its counterpart $g^{\prime}$ in $G^{\prime}$ with $V_{G}(g)$ equal to $V_{G^{\prime}}\left(g^{\prime}\right) \cup\{u, v\}$ if $f=g, V_{G^{\prime}}\left(g^{\prime}\right) \cup\{u\}$ if $g \in F_{G}(u)-\{f\}, V_{G^{\prime}}\left(g^{\prime}\right) \cup\{v\}$ if $g \in F_{G}(v)-\{f\}$, and $V_{G^{\prime}}\left(g^{\prime}\right)$ otherwise. Considering the particular choice of the colors of $u$ and $v$ in either case, it is straightforward that the spv-condition holds for every face of $G$.

Let $f$ be a face of the graph $G$. The number of high-degree vertices (i.e., vertices of degree at least 3) on the boundary of $f$ is called the weight of $f$ and is denoted by $w(f)$. The face $f$ is a pseudodigon if $w(f)=2$ and $f$ is not a digon. We say that $f$ is small if $w(f)<20$, and large if $w(f) \geq 20$. The configuration of a vertex $v$ of $G$ is the tuple obtained by ordering the elements from the multiset $\{w(g): g \in F(v)\}$ in a nondecreasing manner.

Lemma 2.5. Let $v$ be a vertex of $G$ of degree $k \geq 2$. Let the faces incident with $v$ be denoted by $f_{0}, \ldots, f_{k-1}$ in a clockwise order around $v$. Let $l_{i}=w\left(f_{i}\right)$ for every such face $f_{i}$. The following bounds hold for the face degree of $v$ :

(1) if $k \geq 3$, then $d^{F}(v) \leq 4 \sum_{i=0}^{k-1} l_{i}-5 k-3 \sigma$, where $\sigma$ is the number of all $i=0, \ldots, k-1$ with the property that $l_{i}+l_{i+1} \leq 25$ (indices modulo $k$ );

(2) if $k=2$, then $d^{F}(v) \leq 4\left(l_{0}+l_{1}\right)-6$;

(3) If $k=3$, then the configuration of $v$ is different from $(2,8,19)$ and $(3,8,19)$.

For the $f_{0}$-reduced face degree, we have the following:

(4) if $k \geq 3$, then $d^{F}\left(v, f_{0}\right) \leq 4 \sum_{i=1}^{k-1} l_{i}-5 k+9$;

(5) if $k=3$ and $l_{1}+l_{2} \leq 25$, then $d^{F}\left(v, f_{0}\right) \leq 4\left(l_{1}+l_{2}\right)-9$.

Proof. Let $f_{j}$ be a face of $G$ incident with the vertex $v$. Traversing the boundary cycle of $f_{j}$ clockwise from $v$, let $P_{v}\left(f_{j}\right)$ be the subpath of the boundary cycle starting with the successor of $v$ and ending with the predecessor of the last high-degree vertex before $v$. (See Figure 2.2 for an illustration.) Let $p_{v}\left(f_{j}\right)$ be the number of vertices of $P_{v}\left(f_{j}\right)$. Since each vertex in $N^{F}(v)$ is included in at least one of the paths $P_{v}\left(f_{i}\right)(i=0, \ldots, k-1)$, we have

$$
d^{F}(v) \leq \sum_{i=0}^{k-1} p_{v}\left(f_{i}\right)
$$




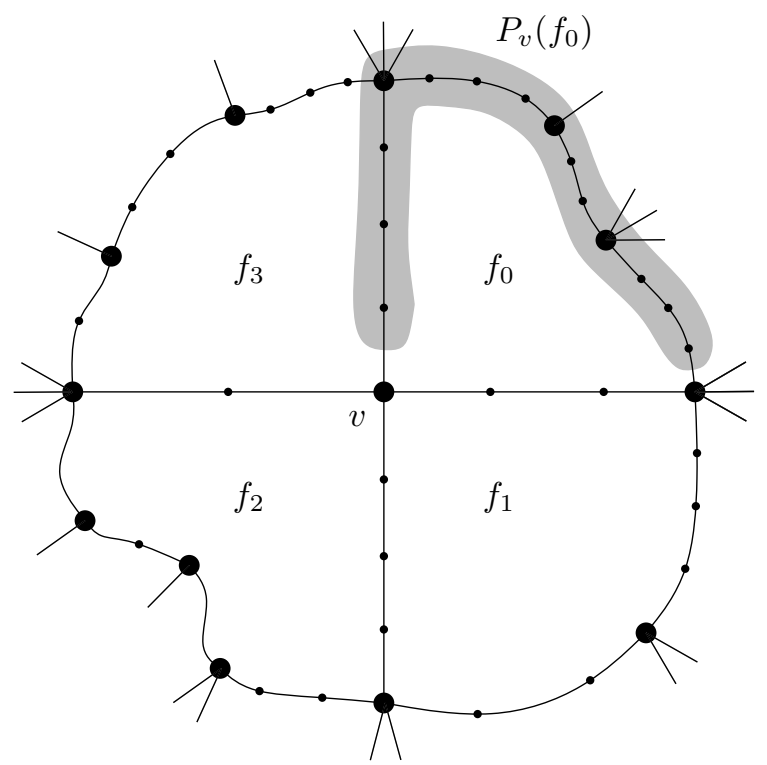

Figure 2.2. The definition of the path $P_{v}\left(f_{i}\right)$ for $i=0$ and a vertex $v$ of degree 4 . Highdegree vertices are shown as larger dots.

We now bound $p_{v}\left(f_{i}\right)$. Assume first that $k \geq 3$. Recall that every two consecutive highdegree vertices on the boundary of $f_{i}$ are separated by at most three vertices of degree 2 (Lemma $2.4(3))$. Since exactly two out of the $l_{i}$ high-degree vertices are excluded from $P_{v}\left(f_{i}\right)$, the path $P_{v}\left(f_{i}\right)$ decomposes into $l_{i}-2$ segments, each consisting of at most three vertices of degree 2 (in $G$ ) followed by a high-degree vertex, and one final segment consisting of up to three vertices of degree 2 and no high-degree vertex. The final segment may be empty. We find:

$$
p_{v}\left(f_{i}\right) \leq 4\left(l_{i}-2\right)+3=4 l_{i}-5 .
$$

We also observe an improvement in the following special case. Let us call the path $P_{v}\left(f_{i}\right)$ deficient if it starts or ends with a high-degree vertex (of $G$ ), or if it contains two consecutive high-degree vertices. In that case, one of the above defined segments contains zero instead of three vertices of degree 2 , and we obtain:

$$
p_{v}\left(f_{i}\right) \leq 4 l_{i}-8 \text { if } P_{v}\left(f_{i}\right) \text { is deficient. }
$$

If $k=2$, analogous reasoning yields

$$
p_{v}\left(f_{i}\right) \leq \begin{cases}4 l_{i}-9 & \text { if } P_{v}\left(f_{i}\right) \text { contains three consecutive high-degree vertices, } \\ 4 l_{i}-7 & \text { if } P_{v}\left(f_{i}\right) \text { starts with two high-degree vertices, } \\ 4 l_{i}-5 & \text { if } P_{v}\left(f_{i}\right) \text { ends at a high-degree vertex } \\ 4 l_{i}-4 & \text { if } P_{v}\left(f_{i}\right) \text { is deficient } \\ 4 l_{i}-2 & \text { otherwise. }\end{cases}
$$


We can now prove part (2) of the lemma. It may be assumed that $P_{v}\left(f_{0}\right)$ starts with a high-degree vertex, since adjacent 2-vertices have the same face degree. By (2.2) and (2.5),

$$
d^{F}(v) \leq p_{v}\left(f_{0}\right)+p_{v}\left(f_{1}\right) \leq\left(4 l_{0}-4\right)+\left(4 l_{1}-2\right)=4\left(l_{0}+l_{1}\right)-6 .
$$

Next, we derive part (1). From (2.2) and (2.3), it follows that

$$
d^{F}(v) \leq 4 \sum_{i=0}^{k-1} l_{i}-5 k \text {. }
$$

We need to improve this estimate by $3 \sigma$, where $\sigma$ is as defined in the lemma. Let $i$ be such that $l_{i}+l_{i+1} \leq 25$ (indices modulo $k$ ) and let $w$ be the first vertex of $P_{v}\left(f_{i+1}\right)$. If the degree of $w$ is 2 , then by part (2) of the lemma, $d^{F}(w) \leq 94$, contradicting Lemma 2.4 (4). Thus, $P_{v}\left(f_{i+1}\right)$ starts with a high-degree vertex and we can apply (2.4) in place of (2.3) to bound $p_{v}\left(f_{i+1}\right)$. This results in an improvement to the upper bound in (2.6) by 3 for each $i$ satisfying $l_{i}+l_{i+1} \leq 25$, and hence in an improvement by $3 \sigma$ in total. Part (1) of the lemma follows.

Let us proceed to part (3). First, note that $\left\{l_{0}, l_{1}, l_{2}\right\} \neq\{2,8,19\}$ by part (1): indeed, we would have

$$
d^{F}(v) \leq 4 \cdot\left(l_{0}+l_{1}+l_{2}\right)-5 \cdot 3-3 \cdot 2=95,
$$

contradicting Lemma 2.4 (4).

Suppose then that $l_{0}=3, l_{1}=8$ and $l_{2}=19$. No vertex of degree 2 in $G$ is incident with both $f_{0}$ and $f_{2}$, since part (2) of the lemma would imply that its face degree is bounded by $4(3+19)-6=82$, contradicting Lemma $2.4(4)$. Thus, $P_{v}\left(f_{0}\right)$ starts with a high-degree vertex, and the same can be proved for $P_{v}\left(f_{1}\right)$ by an identical argument.

We claim that no vertex of degree 2 in $G$ is incident with both $f_{1}$ and $f_{2}$. Suppose to the contrary that there is such a vertex, and let $z$ be the last 2-vertex encountered on the clockwise boundary cycle of $f_{1}$ before $v$. The fact that $P_{v}\left(f_{0}\right)$ starts with a high-degree vertex implies that $P_{z}\left(f_{2}\right)$ ends at a high-degree vertex. Furthermore, since $P_{v}\left(f_{1}\right)$ starts with a high-degree vertex, $P_{z}\left(f_{1}\right)$ either starts with two consecutive high-degree vertices (if $z$ is a neighbor of $v$ ) or contains three consecutive high-degree vertices. By (2.5),

$$
d^{F}(z) \leq\left(4 l_{1}-7\right)+\left(4 l_{2}-5\right)=96
$$

a contradiction with Lemma 2.4 (4). It follows that besides $P_{v}\left(f_{0}\right)$ and $P_{v}\left(f_{1}\right)$, the path $P_{v}\left(f_{2}\right)$ is also deficient. By $(2.4)$,

$$
d^{F}(v) \leq 4 \cdot\left(l_{0}+l_{1}+l_{2}\right)-3 \cdot 8=96,
$$

which again contradicts Lemma 2.4 (4).

We turn to part (4) of the lemma. Unlike the situation in the proof of part $(1), N^{F}\left(v, f_{0}\right)$ is not entirely covered by the sets $V\left(P_{v}\left(f_{i}\right)\right)$, where $i=1, \ldots, k-1$. On the other hand, only a few vertices of $N^{F}\left(v, f_{0}\right)$ are missing in the union of these sets, namely the vertices on the boundary of $f_{k-1}$ not contained in $P_{v}\left(f_{k-1}\right)$. If we let $u$ denote the vertex that 
follows $P_{v}\left(f_{k-1}\right)$ on the boundary of $f_{k-1}$, then the missing vertices are $u$ and the (up to three) vertices of degree 2 following $u$. Consequently,

$$
d^{F}\left(v, f_{0}\right) \leq \sum_{i=1}^{k-1} p_{v}\left(f_{i}\right)+4
$$

and by $(2.3)$,

$$
d^{F}\left(v, f_{0}\right) \leq 4 \sum_{i=1}^{k-1} l_{i}-5(k-1)+4=4 \sum_{i=1}^{k-1} l_{i}-5 k+9 .
$$

Part (5) follows easily from (2.7) using the estimate (2.3) for the face $f_{1}$ and the estimate (2.4) for $f_{2}$ (note that $l_{1}+l_{2} \leq 25$ implies that $P_{v}\left(f_{2}\right)$ is deficient, just as in the proof of (1)). The proof is thus complete.

\subsection{Discharging}

Having explored the properties of the graph $G$, we are ready to use the discharging method to arrive at a contradiction.

We assign an initial charge to the vertices and faces of $G$ as follows:

- each vertex $v$ receives $d(v)-6$ units of charge;

- each face $f$ receives $2|V(f)|-6$ units of charge.

The following observation is a well-known consequence of Euler's formula.

Observation 2.6. The sum of the charges defined above is -12 .

In the first phase, we redistribute the charges according to Rules $1-2$ :

Rule 1. Every face that is not a pseudodigon sends two units of charge to each incident 2-vertex. Each pseudodigon does the same, except that one of the respective 2-vertices receives no charge.

Observe that after the application of Rule 1 , the charge of each face is nonnegative. In addition, the charge of every large face is at least $2 \cdot 20-6=34$.

Rule 2. Every small face distributes its remaining charge evenly to all incident high-degree vertices (i.e., vertices of degree at least 3). Each large face (i.e., a face of weight at least 20) behaves in the same way, except that it retains a charge of 4.

After applying the above rules the first phase is completed. In the second phase, Rule 3 is applied to the vertices that ended up with negative charge after the first phase.

Rule 3. If a vertex has a negative charge of $c$ and is incident with a large face $f$, then it receives the charge of $-c$ from $f$. 
We will show that the final charge of every vertex and face in $G$ is nonnegative, contradicting Observation 2.6.

Recall from Subsection 2.1 that the configuration of a vertex $v$ of $G$ is obtained by ordering the multiset $\{w(g): g \in F(v)\}$ in a nondecreasing way. If we remove (one copy of) the element $w(f)$ from this ordered multiset, we obtain the $f$-reduced configuration of $v$.

It will be convenient to alter the definition of the weight of a face $f$ and the configuration of a vertex $v$ as follows. The modified weight $w^{\prime}(f)$ of $f$ is defined as 3 if $w(f)=2$, and $w(f)$ otherwise. Replacing the weight of each face by its modified weight in the definition of the configuration of $v$, we obtain the modified configuration of $v$. The modified $f$-reduced configuration of $v$ is obtained from the $f$-reduced configuration of $v$ in an analogous way.

First we analyze how much charge a vertex $v$ of high degree $d$ receives by Rule 2 . Denote the faces incident with $v$ by $f_{i}, i=0, \ldots, d-1$, and let $n_{i}$ be the number of 2-vertices incident with $f_{i}$. After applying Rule 1 , each $f_{i}$ has charge $2\left|V\left(f_{i}\right)\right|-6-2 n_{i}$ if $f_{i}$ is not a pseudodigon, and $2\left|V\left(f_{i}\right)\right|-6-2\left(n_{i}-1\right)$ otherwise. By Lemma $2.4(2), f_{i}$ is not a digon so in both cases the charge can be written as $2 w^{\prime}\left(f_{i}\right)-6$. Hence, when $f_{i}$ is a small face, it sends $v$ the charge of

$$
\frac{2 w^{\prime}\left(f_{i}\right)-6}{w\left(f_{i}\right)}=2-\frac{6}{w^{\prime}\left(f_{i}\right)} .
$$

(The equality is true as $w\left(f_{i}\right)=w^{\prime}\left(f_{i}\right)$ if $f_{i}$ is not a pseudodigon, and $2 w^{\prime}\left(f_{i}\right)-6=0$ otherwise.) On the other hand, when $f_{i}$ is a large face, it sends $v$

$$
\frac{2 w^{\prime}\left(f_{i}\right)-6-4}{w\left(f_{i}\right)}=2-\frac{10}{w^{\prime}\left(f_{i}\right)}
$$

units of charge. Note that in both cases the charge received by $v$ from $f_{i}$ is nonnegative. In total, the vertex $v$ obtains the nonnegative charge of

$$
\begin{gathered}
\sum_{\substack{i \\
w^{\prime}\left(f_{i}\right)<20}}\left(2-\frac{6}{w^{\prime}\left(f_{i}\right)}\right)+\sum_{\substack{i \\
w^{\prime}\left(f_{i}\right) \geq 20}}\left(2-\frac{10}{w^{\prime}\left(f_{i}\right)}\right) \\
=2 d-6 \sum_{\substack{i \\
w^{\prime}\left(f_{i}\right)<20}} \frac{1}{w^{\prime}\left(f_{i}\right)}-10 \sum_{\substack{i \\
w^{\prime}\left(f_{i}\right) \geq 20}} \frac{1}{w^{\prime}\left(f_{i}\right)} .
\end{gathered}
$$

Next, we establish the following two essential claims. For convenience, we refer to the vertices with a negative charge after the first phase as special vertices. Since the initial charge of a vertex $v$ is $d(v)-6$ and each vertex receives a nonnegative charge during the application of Rule 2, every special vertex has degree at most 5 .

Claim 1. Every special vertex is incident with a large face.

Proof. We proceed by contradiction, assuming that $v$ is a special vertex not incident with any large face. First suppose that $d(v)=2$; let $f_{1}$ and $f_{2}$ be the two faces incident with 
$v$. As the initial charge of $v$ is -4 , at least one of these faces, say $f_{1}$, is a pseudodigon by Rule 1. By assumption, $w\left(f_{2}\right) \leq 19$. Hence $d^{F}(v) \leq 78$ by Lemma 2.5 (2), a contradiction to Lemma $2.4(4)$.

Therefore, let $v$ be a special vertex of high degree $d$. Summing its initial charge and the charge (2.8) received by Rule 2 gives

$$
3 d-6-6 \sum_{i} \frac{1}{w^{\prime}\left(f_{i}\right)}<0,
$$

or equivalently,

$$
\sum_{i} \frac{1}{w^{\prime}\left(f_{i}\right)}>\frac{d}{2}-1 .
$$

We proceed by case analysis; let $\left(l_{i}^{\prime}\right)$ denote the modified configuration of $v$. (We write $\left(l_{i}^{\prime}\right)$ instead of $\left(l_{i}\right)$ as a reminder that the configuration is a modified one.) Assume first that $v$ is of degree 3 . Then $l_{0}^{\prime} \leq 5$, otherwise (2.9) fails since its left hand side is at most $3 \cdot(1 / 6)$ which equals its right hand side $1 / 2$. Since $v$ is not incident with any large face, we have $l_{1}^{\prime}, l_{2}^{\prime} \leq 19$. We aim to use Lemma 2.5 to bound $d^{F}(v)$. Although it is formulated for ordinary (non-modified) configurations, the monotonicity of the upper bounds ensures that the lemma remains valid if the configuration is a modified one. By Lemma 2.5 (1), $d^{F}(v) \leq 4 \sum_{i} l_{i}^{\prime}-21$. Consequently, Lemma 2.4 (4) implies that

$$
\sum_{i} l_{i}^{\prime} \geq 30
$$

If $l_{0}^{\prime}=3$ and $l_{1}^{\prime} \leq 9$, then by $(2.10)\left(l_{i}^{\prime}\right)$ is one of the three tuples $(3,8,19),(3,9,18)$, and $(3,9,19)$. The first of these is excluded by Lemma $2.5(3)$, and the remaining two contradict (2.9). If $l_{0}^{\prime}=3$ and $l_{1}^{\prime}>9$, then by Lemma 2.3 applied to the tuples $(3,10,15)$ and $\left(l_{i}^{\prime}\right)$, in this order, either $\sum_{i} l_{i}^{\prime} \leq 28$ or $\sum_{i} 1 / l_{i}^{\prime} \leq 1 / 2$. However, that contradicts (2.10) or (2.9), respectively.

Hence $l_{0}^{\prime} \geq 4$. If $l_{0}^{\prime}, l_{1}^{\prime}=4$, then $\sum_{i} l_{i}^{\prime} \leq 27$, which is impossible by (2.10). Otherwise we may use Lemma 2.3 for the tuples $(4,5,20)$ and $\left(l_{i}^{\prime}\right)$, and obtain a contradiction to $(2.10)$ or (2.9) again.

Thus $d \geq 4$; as we have remarked above, $d \leq 5$. Lemmas 2.5 (1) and 2.4 (4) imply (2.10) again. In particular, $\left(l_{i}^{\prime}\right)$ cannot be of the form $(3,3,3, x)$. If $d=4$, then by Lemma 2.3 applied to the tuple $(3,3,4,12), \sum_{i} l_{i}^{\prime} \leq 22$ or $\sum_{i} 1 / l_{i}^{\prime} \leq 1$, contradicting (2.10) or (2.9). For $d=5$, we obtain a similar contradiction by applying Lemma 2.3 to $(3,3,3,3,6)$, which yields $\sum_{i} l_{i}^{\prime} \leq 18$ or $\sum_{i} 1 / l_{i}^{\prime} \leq 3 / 2$.

Claim 2. Every large face has a nonnegative final charge.

Proof. Let $f$ be an arbitrary large face of $G$. We start by listing the possible $f$-reduced or modified $f$-reduced configurations of special vertices incident with $f$, and for each case we note a lower bound on the charge of these vertices. Take such a special vertex $v$. 
If $v$ is a 2 -vertex, then by Rule 1 its $f$-reduced configuration is (2) and the charge equals -2 .

Now suppose that $v$ is of high degree $d$. Let $\left(l_{i}^{\prime}\right), i=1, \ldots, d-1$, be its modified $f$-reduced configuration, and let $d^{\prime}$ denote the number of large faces incident with $v$. As noted earlier, $d \leq 5$. By considering the initial charge of $v$ and (2.8), we see that after applying Rule 2, $v$ has charge

$$
3 d-6-6 \sum_{\substack{i \\ w^{\prime}\left(f_{i}\right)<20}} \frac{1}{w^{\prime}\left(f_{i}\right)}-10 \sum_{\substack{i \\ w^{\prime}\left(f_{i}\right) \geq 20}} \frac{1}{w^{\prime}\left(f_{i}\right)}
$$

As the charge is negative by assumption,

$$
3 d-6-6 \sum_{\substack{i \\ w^{\prime}\left(f_{i}\right)<20}} \frac{1}{w^{\prime}\left(f_{i}\right)}-\frac{d^{\prime}}{2}<0
$$

by the definition of large face. Furthermore, $w^{\prime}\left(f_{i}\right) \geq 3$ always, and hence

$$
3 d-6-2\left(d-d^{\prime}\right)-d^{\prime} / 2<0
$$

Since the left hand side equals $d-6+3 d^{\prime} / 2$, we deduce that $d^{\prime}=1$ (i.e., $f$ is the only large face incident with $v$ ), and that $d \leq 4$.

Assume first that $d=3$. Then (2.11) reduces to

$$
\frac{5}{2}-6\left(\frac{1}{l_{1}^{\prime}}+\frac{1}{l_{2}^{\prime}}\right)<0 .
$$

We infer that either $l_{1}^{\prime}=3$ and $l_{2}^{\prime} \leq 11$, or $l_{1}^{\prime}=4$ and $l_{2}^{\prime} \leq 5$. The charge of $v$ is at least $1 / 2-6 / l_{2}^{\prime}$ in the former, and at least $1-6 / l_{2}^{\prime}$ in the latter case.

Now let $d=4$. By (2.11),

$$
\frac{11}{2}-6 \sum_{i} \frac{1}{l_{i}^{\prime}}<0
$$

If some $l_{i}^{\prime}$ were greater than or equal to 4 , this inequality would not hold. Hence $\left(l_{i}^{\prime}\right)=$ $(3,3,3)$ and the charge of $v$ is at least $-1 / 2$. We summarize the results in Table 1.

Let $S$ denote the set of all special vertices incident with $f$ and $R$ their total charge after the completion of the first phase. We observe the following:

Any two vertices $u, v \in S$ have at least two common incident faces.

Suppose the contrary. In view of the possible $f$-reduced or modified $f$-reduced configurations of $u$ and $v$ listed in Table 1, Lemma 2.5 (4) and (5) implies that both $d^{F}(u, f)$ and $d^{F}(v, f)$ are at most 47. On the other hand, Lemma 2.4 (5) and the assumption that $f$ is the only face incident with both $u$ and $v$ imply that $d^{F}(u, f)+d^{F}(v, f)>95$, a contradiction. 


\begin{tabular}{|l|l|}
\hline (modified) $f$-reduced configuration & charge \\
\hline$(2)$ & -2 \\
$(3, x), x \leq 11$ & $\geq 1 / 2-6 / x \geq-3 / 2$ \\
$(4, x), x \leq 5$ & $\geq 1-6 / x \geq-1 / 2$ \\
$(3,3,3)$ & $\geq-1 / 2$ \\
\hline
\end{tabular}

Table 1. The proof of Claim 2: the list of possible $f$-reduced (the first line) or modified $f$-reduced (the other lines) configurations of special vertices incident with the face $f$, together with the charge of these vertices.

We proceed to prove Claim 2 by contradiction. Suppose that $f$ has a negative charge after the application of Rule 3. Since the charge of $f$ after the first phase is 4 units (by Rule 2 for large faces), this is equivalent to the condition

$$
R<-4 \text {. }
$$

Considering the lower bounds for charges in Table 1, we see that there are at least three special vertices.

Let $v \in S$ be a 2-vertex; the other face $f^{\prime}$ incident with $v$ is a pseudodigon. By Rule 1 and (2.12), every other vertex $v^{\prime}$ in $S$ is one of the two high-degree vertices $v_{1}, v_{2}$ incident with $f^{\prime}$. Therefore $S=\left\{v, v_{1}, v_{2}\right\}$; it follows that $v_{1}$ and $v_{2}$ are both of degree 3 by assumption (2.13). This means that $F\left(v_{1}\right)=F\left(v_{2}\right)$, and hence the configuration of both vertices is the same. Considering (2.13) again, the modified $f$-reduced configuration of both $v_{1}$ and $v_{2}$ is $(3,3)$.

At this point, we digress by making an auxiliary observation:

Let $g$ be a face of $G$ with $w(g) \leq 3$. The intersection of the boundaries of $f$ and $g$ consists of pairwise disjoint paths, of which at most one is nontrivial; the internal vertices of all these paths are of degree 2 in $G$. Furthermore, any two vertices of degree 3 incident with both $f$ and $g$ are precisely the end-vertices of such a nontrivial path, and hence, there are at most two such vertices.

To prove this, let us denote the intersection of the boundaries of $f$ and $g$ by $H$. The assertion about the structure of $H$ is obvious by considering the 2-connectedness of $G$ and the weights of both $f$ and $g$. Let $\mathcal{P}$ denote the set of the respective paths. If $v$ is a vertex of degree 3 incident with $f$ and $g$, then $H$ must contain an edge incident with $v$, i.e., $v$ lies on - and hence is an end-vertex of - a nontrivial path in $\mathcal{P}$. The second assertion easily follows.

Applying (2.14) to the face incident with $v_{1}$ different from $f$ and $f^{\prime}$, and recalling that $f^{\prime}$ is a pseudodigon, we infer that $w(f)=2$; a contradiction.

Thus all vertices in $S$ are of high degree. Suppose that $S=\left\{v_{1}, v_{2}, v_{3}\right\}$. Then by assumption (2.13), the modified $f$-reduced configuration of $v_{1}, v_{2}$, and $v_{3}$ is $(3,3)$. Hence 
by (2.14), no face other than $f$ is incident with the three vertices. By (2.12), $G$ contains three different faces $f_{i j}, 1 \leq i<j \leq 3$, incident with both $v_{i}$ and $v_{j}$ and different from $f$. By (2.14), the boundary of $f$ is precisely $\cup \mathcal{P}$; thus $w(f)=3$, a contradiction to the assumption that $f$ is large.

Therefore $|S| \geq 4$. We claim that $G$ contains a face $f^{\prime} \neq f$ incident with all the vertices in $S$. To prove this, consider three vertices $u, x, v$ in $S$ consecutively encountered on a facial walk of $f$. By (2.12), there is a curve $\mathcal{C}_{u v}$ connecting $u$ and $v$ through a face $f_{u v} \neq f$ of $G$. Consider any vertex $y \in S-\{u, x, v\}$. There is a curve $\mathcal{C}_{x y}$ connecting $x$ and $y$ through a face $f_{x y} \neq f$. If we let $\mathcal{C}_{v u}$ be a curve connecting $v$ to $u$ through $f$, then $\mathcal{C}_{u v} \cup \mathcal{C}_{v u}$ is a closed curve separating $x$ from $y$. Consequently, $\mathcal{C}_{u v}$ intersects $\mathcal{C}_{x y}$, and therefore $y$ is incident with the face $f_{u v}$. The assertion easily follows.

Now let $k:=|S|$. Then $w\left(f^{\prime}\right) \geq k$, and consequently the $f$-reduced configuration of each $v$ in $S$ contains a number greater than or equal to $k$. From Table 1 , we see that

$$
R \geq k\left(\frac{1}{2}-\frac{6}{k}\right)=\frac{k}{2}-6
$$

the right side of which is at least -4 by the condition on $k$. This contradicts assumption (2.13).

With the help of the two preceding claims, we can easily finish the proof. By Claim 1 and Rule 3, every special vertex - and hence every vertex - ends up with a nonnegative charge. The final charge of every face is nonnegative as well; Rule 2 and Claim 2 guarantee this for small and large faces respectively. However, as already mentioned, this contradicts Observation 2.6.

\section{Lower bound}

In this section, we provide examples showing that the best possible constant bound on $\chi_{s}$ for the class of 2-connected plane simple graphs is at least 8 , and the corresponding bound for proper spv-colorings is at least 10. Note that for the class of 2-connected plane (multi)graphs, the latter example implies a lower bound of 10 for general spv-colorings. Indeed, if we replace each of its edges by a digon bounding a face, then every spv-coloring of the resulting graph is necessarily a proper spv-coloring of the original simple graph.

First, we focus on the bound for proper spv-colorings. We construct a graph $G_{55}$ on ten vertices by linking two disjoint cycles $C^{1}, C^{2}$ on five vertices with two additional edges whose endvertices in each cycle are adjacent. See Figure 3.1 (a).

By the spv-conditions for the two faces of $G_{55}$ of length 5 , every proper spv-coloring $c$ must assign each vertex of $C^{1}$ a different color; the same holds for $C^{2}$. The spv-condition for the face of $G_{55}$ of length 10 then implies that $c$ uses each color precisely once.

Second, we consider the bound on $\chi_{s}$ (where the coloring is allowed to be improper). Take a three-sided prism $G_{3}$ embedded in the plane so that one of its triangular faces is the outer face $f_{3}$. As observed by Czap and Jendrol' [3, proof of Lemma 5.1], every coloring 


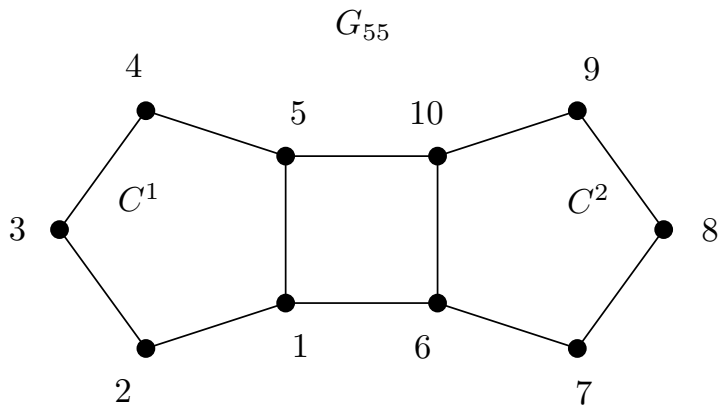

(a) The graph $G_{55}$.
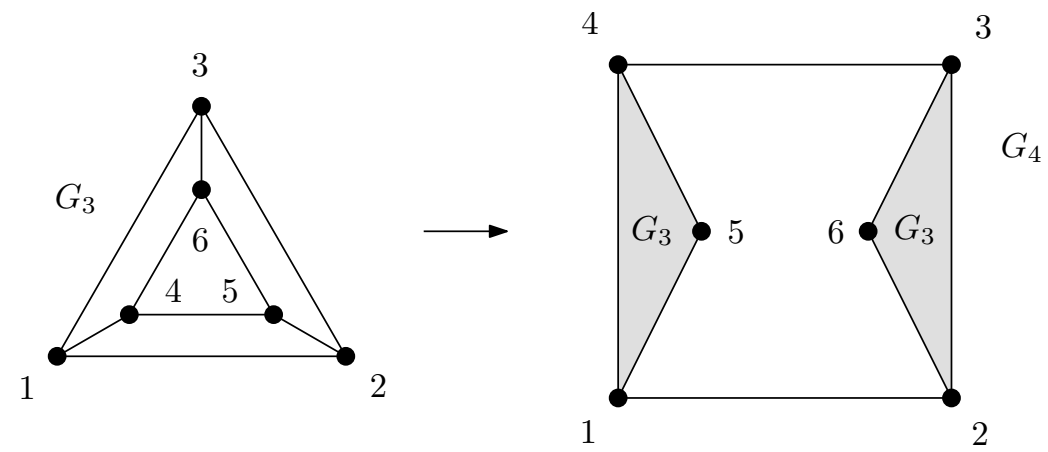

(b) The graphs $G_{3}$ (left) and $G_{4}$ (right).

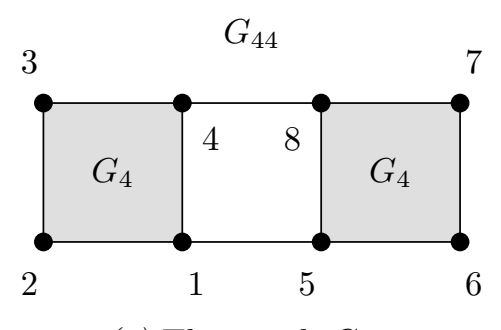

(c) The graph $G_{44}$.

Figure 3.1. Illustrations for Section 3. The labeled gray areas represent the respective subgraphs not depicted in detail. For each graph, the relevant coloring is unique up to symmetry; it is indicated by numerical labels. 
$c$ of $G_{3}$ such that every face of $G_{3}$ distinct from $f_{3}$ satisfies the spv-condition colors each boundary vertex of $f_{3}$ with a different color.

Now construct a graph $G_{4}$ from a cycle on four vertices by replacing every other edge with a copy of $G_{3}$ in such a way that the outer face $f_{4}$ of $G_{4}$ is of length 4 ; see Figure 3.1 (b). Let $c^{\prime}$ be a coloring of $G_{4}$ satisfying the spv-condition for each face of $G_{4}$ other than $f_{4}$. When restricted to the vertices of any of the copies of $G_{3}, c^{\prime}$ has the property of the coloring $c$ discussed above. This and the spv-condition for the face of $G_{4}$ of length 6 imply that $c^{\prime}$ assigns a different color to each boundary vertex of $f_{4}$.

Finally, we reproduce the construction of $G_{55}$ with copies of $G_{4}$ in place of the cycles on five vertices. Thereby we obtain a graph $G_{44}$ with the outer face $f_{44}$ of length 8 , such that every spv-coloring of $G_{44}$ is injective on $V\left(f_{44}\right)$. The graph $G_{44}$ is shown in Figure 3.1 (c).

\section{References}

[1] D. P. Bunde, K. Milans, D. B. West, and H. Wu, Parity and strong parity edge-coloring of graphs, Congr. Numer. 187 (2007) 193-213.

[2] D. P. Bunde, K. Milans, D. B. West, and H. Wu, Optimal strong parity edge-coloring of complete graphs, Combinatorica 28 (2008) 625-632.

[3] J. Czap and S. Jendrol', Colouring vertices of plane graphs under restrictions given by faces, Discuss. Math. Graph Theory 29 (3) (2009) 521-543.

[4] J. Czap, S. Jendrol', and M. Voigt, Parity vertex colouring of plane graphs, Discrete Math. 311 (6) (2011) 512-520.

[5] J. Czap, S. Jendrol', F. Kardoš and R. Soták, Facial parity edge colouring of plane pseudographs, Discrete Math. 312 (17) (2012) 2735-2740.

[6] R. Diestel, Graph Theory, Springer-Verlag, Heidelberg, 2010. 